K. YANO

KODAI MATH. J.

1 (1978), 30-44

\title{
INFINITESIMAL VARIATIONS OF SUBMANIFOLDS
}

\author{
By Kentaro Yano
}

\section{$\S 0$. Introduction.}

The purpose of the present paper is to study variations of the metric tensor, the Christoffel symbols and the second fundamental tensors of submanifolds under infinitesimal variations of the submanifolds.

The method used here is to displace the deformed quantities back parallelly from the displaced point to the original point and to compare the parallelly displaced back quantities and the original quantities, [3], [4].

In $\S 1$, we state formulas for submanifolds of a Riemannian manifold needed for the later discussions including equations of Gauss, Codazzi and Ricci. [1].

In $\S 2$, we consider infinitesimal variations of submanifolds of a Riemannian manifold. We define parallel variations of submanifolds and study their properties.

$\S 3$ is devoted to the study of variations of the fundamental metric tensor of the submanifold. We discuss isometric, conformal and volume-preserving. variations.

We study in $\S 4$ the variations of the Christoffel symbols and those of linear connection induced in the normal bundle. When the submanifold is compact or complete and irreducible, we obtain some global results.

In the last $\S 5$, we study variations of the second fundamental tensors and prove some global propositions. (For normal variations, see [2]).

\section{$\S 1$. Preliminaries.}

Let $M^{m}$ be an $m$-dimensional Riemannian manifold covered by a system of coordinate neighborhoods $\left\{U ; x^{h}\right\}$ and denote by $g_{j i}, \Gamma_{j i}^{h}, \nabla_{\jmath}, K_{k j i}{ }^{h}$ and $K_{j i}$ the metric tensor, the Christoffel symbols formed with $g_{j i}$, the operator of covariant differentiation with respect to $\Gamma_{j i}^{h}$, the curvature tensor and the Ricci tensor of $M^{m}$ respectively, where and in the sequel the indices $h, i, j, k, \cdots$ run over the range $\{\overline{1}, \overline{2}, \cdots, \bar{m}\}$.

Let $M^{n}$ be an $n$-dimensional Riemannian manifold covered by a system of coordinate neighborhoods $\left\{V ; y^{a}\right\}$ and denote by $g_{c b}, \Gamma_{c b}^{a}, \nabla_{c}, K_{d c b}{ }^{a}$ and $K_{c b}$ the corresponding quantities of $M^{n}$ respectively, where and in the sequel the indices

Received February 20, 1976 
$a, b, c, d, \cdots$ run over the range $\{1,2, \cdots, n\}$.

We suppose that $M^{n}$ is isometrically immersed in $M^{m}$ by the immersion $\imath$ : $M^{n} \rightarrow M^{m}$ and identify $i\left(M^{n}\right)$ with $M^{n}$. We represent the immersion by

$$
x^{h}=x^{h}\left(y^{a}\right)
$$

and put

$$
B_{b}{ }^{h}=\partial_{b} x^{h}, \quad\left(\partial_{b}=\partial / \partial y^{b}\right) .
$$

Then $B_{b}{ }^{h}$ are $n$ linearly independent vectors of $M^{m}$ tangent to $M^{n}$. Since the immersion is isometric, we have

$$
g_{c b}=B_{c b}^{j i} g_{j i},
$$

where $B_{c b}^{j i}=B_{c}{ }^{j} B_{b}{ }^{2}$. We denote by $C_{y}{ }^{h} m-n$ mutually orthogonal unit normals to $M^{n}$, where and in the sequel the indices $x, y, z$ run over the range $\{n+1, n+2$, $\cdots, m\}$. Then the metric tensor of the normal bundle of $M^{n}$ is given by

$$
g_{z}=C_{z}{ }^{3} C_{y}{ }^{2} g_{j i}
$$

and has values $g_{z y}=\delta_{z y}, \delta_{z y}$ denoting the Kronecker delta.

It is well known that $\Gamma_{c b}^{a}$ and $\Gamma_{j i}^{h}$ are related by

$$
\Gamma_{c b}^{a}=\left(\partial_{c} B_{b}{ }^{h}+\Gamma_{j i}^{h} B_{c b}^{j i}\right) B^{a}{ }_{h},
$$

where $B^{a}{ }_{h}=B_{b}{ }^{2} g^{b a} g_{i h}, g^{b a}$ being contravariant components of the metric tensor $g_{c b}$ of $M^{n}$ and the components $\Gamma_{c y}^{x}$ of the connection induced in the normal bundle are given by

$$
\Gamma_{c y}^{x}=\left(\partial_{c} C_{y}{ }^{h}+\Gamma_{j i}^{h} B_{c}{ }^{\jmath} C_{y}{ }^{i}\right) C^{x}{ }_{h},
$$

where $C^{x}{ }_{h}=C_{y}{ }^{2} g^{y x} g_{i n}, g^{y x}$ being contravariant components of the metric tensor $g_{y x}$ of the normal bundle.

If we denote by $\nabla_{c} B_{b}{ }^{h}$ and $\nabla_{c} C_{y}{ }^{h}$ the van der Waerden-Bortolotti covariant derivatives of $B_{b}{ }^{h}$ and $C_{y}{ }^{h}$ along the $M^{n}$ respectively, that is, if we put

$$
\nabla_{c} B_{b}{ }^{h}=\partial_{c} B_{b}{ }^{h}+\Gamma_{j l}^{h} B_{c b}^{j i}-\Gamma_{c b}^{a} B_{a}{ }^{h}
$$

and

$$
\nabla_{c} C_{y}{ }^{h}=\partial_{c} C_{y}{ }^{h}+\Gamma_{j i}^{h} B_{c}{ }^{\jmath} C_{y}{ }^{2}-\Gamma_{c y}^{x} C_{x}{ }^{h},
$$

then we can write equations of Gauss and those of Weingarten in the form

$$
\begin{gathered}
\nabla_{c} B_{b}{ }^{h}=h_{c b}{ }^{x} C_{x}{ }^{h}, \\
\nabla_{c} C_{y}{ }^{h}=-h_{c}{ }^{a}{ }_{y} B_{a}{ }^{h}
\end{gathered}
$$

respectively, where $h_{c b}{ }^{x}$ are the second fundamental tensors of $M^{n}$ with respect to the normals $C_{x}{ }^{h}$ and $h_{c}{ }^{a}{ }_{x}=h_{c b x} g^{b a}=h_{c b}{ }^{y} g_{y x} g^{b a}$.

Equations of Gauss, Codazzi and Ricci are respectively 


$$
\begin{gathered}
K_{d c b}{ }^{a}=K_{k j i}{ }^{h} B_{d c b h}^{k j i a}+h_{d}{ }^{a}{ }_{x} h_{c b}{ }^{x}-h_{c}{ }^{a}{ }_{x} h_{d b}{ }^{x}, \\
0=K_{k j i}{ }^{h} B_{d c b}^{k j i} C^{x}{ }_{h}-\left(\nabla_{d} h_{c b}{ }^{x}-\nabla_{c} h_{d b}{ }^{x}\right)
\end{gathered}
$$

and

$$
K_{d c y}{ }^{x}=K_{k j i}{ }^{h} B_{d c}^{k j} C_{y}{ }^{2} C^{x}{ }_{n}+\left(h_{d e}{ }^{x} h_{c}{ }^{e} y-h_{c e}{ }^{x} h_{d}{ }^{e} y\right),
$$

where $B_{d c b h}^{b j i a}=B_{d}{ }^{k} B_{c}{ }^{j} B_{b}{ }^{2} B^{a}{ }_{h}, B_{d c b}^{b j j}=B_{d}{ }^{k} B_{c}{ }^{j} B_{b}{ }^{2}$ and $K_{d c y}{ }^{x}$ is the curvature tensor of the connection induced in the normal bundle.

\section{§ 2. Infinitesimal variations.}

We now consider a variation of $M^{n}$ in $M^{m}$ given by

$$
\bar{x}^{h}=x^{h}+\xi^{h}(y) \varepsilon,
$$

where $g_{{ }_{i}} \xi^{\jmath} \xi^{\imath}>0$ and $\varepsilon$ is an infinitesimal. We then have

$$
\bar{B}_{b}{ }^{h}=B_{b}{ }^{h}+\left(\partial_{b} \xi^{h}\right) \varepsilon,
$$

where $\bar{B}_{b}{ }^{h}=\partial_{b} \bar{x}^{h}$ are $n$ linearly independent vectors tangent to the deformed submanifold at the deformed point $\left(\bar{x}^{h}\right)$.

If we displace $\bar{B}_{b}{ }^{h}$ back parallelly from the point $\left(\bar{x}^{h}\right)$ to $\left(x^{h}\right)$, we obtain

$$
\widetilde{B}_{b}{ }^{h}=\bar{B}_{b}{ }^{h}+\Gamma_{j i}^{h}(x+\xi \varepsilon) \xi^{j} \bar{B}_{b}{ }^{2} \varepsilon,
$$

that is,

$$
\tilde{B}_{b}{ }^{h}=B_{b}{ }^{h}+\left(\nabla_{b} \xi^{h}\right) \varepsilon,
$$

neglecting the terms of order higher than one with respect to $\varepsilon$, where

$$
\nabla_{b} \xi^{h}=\partial_{b} \xi^{h}+\Gamma_{j i}^{h} B_{b}{ }^{\jmath} \xi^{\imath} .
$$

In the sequel we always neglect terms of order higher than one with respect to the infinitesimal $\varepsilon$.

Thus putting

$$
\delta B_{b}{ }^{h}=\widetilde{B}_{b}{ }^{h}-B_{b}{ }^{h},
$$

we have

$$
\delta B_{b}{ }^{h}=\nabla_{b} \xi^{h} \varepsilon .
$$

If we put

$$
\xi^{h}=\xi^{a} B_{a}{ }^{h}+\xi^{x} C_{x}{ }^{h},
$$

we have

$$
\begin{aligned}
\nabla_{b} \xi^{h}= & \left(\nabla_{b} \xi^{a}-h_{b}{ }^{a}{ }_{x} \xi^{x}\right) B_{a}{ }^{h} \\
& +\left(\nabla_{b} \xi^{x}+h_{b a}{ }^{x} \xi^{a}\right) C_{x}{ }^{h},
\end{aligned}
$$


and consequently, putting

$$
\begin{aligned}
& \xi_{b}{ }^{a}=\nabla_{b} \xi^{a}-h_{b}{ }^{a} \xi^{x}, \\
& \xi_{b}{ }^{x}=\nabla_{b} \xi^{x}+h_{b a}{ }^{x} \xi^{a},
\end{aligned}
$$

we have

$$
\nabla_{b} \xi^{h}=\xi_{b}{ }^{a} B_{a}{ }^{h}+\xi_{b}{ }^{x} C_{x}{ }^{h} .
$$

From (2.5), (2.6) and (2.11), we have

$$
\tilde{B}_{b}{ }^{h}=\left(\delta_{b}^{x}+\xi_{b}{ }^{a} \varepsilon\right) B_{a}{ }^{h}+\xi_{b}{ }^{x} C_{x}{ }^{h} \varepsilon .
$$

When the tangent space at a point $\left(x^{h}\right)$ of the submanifold and that at the corresponding point $\left(\bar{x}^{h}\right)$ of the deformed submanifold are parallel, we say that the variation is parallel.

From (2.12), we have

PROPOSITION 2.1. In order for a variation of a submanifold to be parallel, it is necessary and sufficient that

$$
\xi_{b}^{x}=\nabla_{b} \xi^{x}+h_{b a}^{x} \xi^{a}=0 .
$$

When $\xi^{x}=0$, that is, when the variation vector $\xi^{h}$ is tangent to the submanifold we say that the variation is tangential and when $\xi^{a}=0$, that is, when the variation vector $\xi^{h}$ is normal to the submanifold we say that the variation is normal.

From Proposition 2.1, we have

Proposition 2.2. In order for a tangential variation of a submanifold to be parallel, it is necessary and sufficient that

$$
h_{b a}^{x} \xi^{a}=0 .
$$

COROLlary 1. A tangential variation of a totally geodesic submantold is always parallel.

COROLlaRY 2. A tangential variation of a totally umbilical submanifold with non-vanishing mean curvature is never parallel.

From Proposition 2.1, we also have

PROPOSITION 2.3. In order for a normal variation of a submanifold to be parallel, it is necessary and sufficient that

$$
\nabla_{b} \xi^{x}=0,
$$

that is, the variation vector $\xi^{x} C_{x}^{h}$ is parallel in the normal bundle.

For a parallel normal variation, we have $\nabla_{b} \xi^{x}=0$, which shows that $\nabla_{b}\left(g_{z y} \xi^{z} \xi^{y}\right)$ $=0$. Thus we have 
COROLlary 1. A parallel normal variation of a submanifold displaces each point of the submanifold the same distance.

When the submanifold is a hypersurface a normal variation is given by $\bar{x}^{h}=x^{h}+\lambda C^{h} \varepsilon, C^{h}$ being the unique unit normal to the hypersurface and $\lambda$ a positive function and consequently (2.15) reduces to $\nabla_{b} \lambda=0$ and we have

PROPOSITION 2.4. In order for a normal variation of a hypersurface to be parallel, it is necessary and sufficient that the normal variation displaces each point of the hypersurface the same distance.

\section{§3. Variations of the metric tensor.}

Now applying the operator $\delta$ to (1.3) and using (2.6), (2.8) and $\delta g_{j i}=0$, we flnd

$$
\delta g_{c b}=\left(\nabla_{c} \xi_{b}+\nabla_{b} \xi_{c}-2 h_{c b x} \xi^{x}\right) \varepsilon,
$$

where $\xi_{b}=g_{b a} \xi^{a}$, from which

$$
\delta g^{b a}=-\left(\nabla^{b} \xi^{a}+\nabla^{a} \xi^{b}-2 h^{b a}{ }_{x} \xi^{x}\right) \varepsilon,
$$

where $\nabla^{b}=g^{b a} \nabla_{a}$ and $h^{b a}{ }_{x}=g^{e b} g^{d a} h_{e d x}$.

A variation of a submanifold for which $\delta g_{c b}=0$ is said to be isometric and that for which $\delta g_{c b}$ is proportional (with constant proportional factor) to $g_{c b}$ is said to be conformal (homothetrc).

From (3.1), we have

PROPOSITION 3.1. In order for a variation of a submanifold to be isometric, it is necessary and sufficient that

$$
\nabla_{c} \xi_{b}+\nabla_{b} \xi_{c}-2 h_{c b x} \xi^{x}=0 .
$$

Proposition 3.2. [5] In order for a tangential variation of a submanifold to be isometric, it is necessary and sufficient that

$$
\mathcal{L} g_{c b}=\nabla_{c} \xi_{b}+\nabla_{b} \xi_{c}=0,
$$

$\mathcal{L}$ denoting the Lie dervative with respect to $\xi^{a}$.

Proposition 3.3. In order for a normal variation of a submanifold to be isometric, it is necessary and·sufficient that

$$
h_{c b x} \xi^{x}=0,
$$

that is, the submanfold is geodesic with respect to the direction of the normal variation.

COROLlary 1. A submanifold is totally geodesic if and only if every normal variation is isometruc. 
From (3.1), we also have

PROPOSITION 3.4. In order for a variation of a submanifold to be conformal (homothetic), it is necessary and sufficient that

$$
\nabla_{c} \xi_{b}+\nabla_{b} \xi_{c}-2 h_{c b x} \xi^{x}=2 \lambda g_{c b}
$$

$\lambda$ being a certain function (constant).

PROPOSITION 3.5. [5] In order for a tangential variation of a submanifold to be conformal (homothetic), it is necessary and sufficient that

$$
\mathcal{L} g_{c b}=\nabla_{c} \xi_{b}+\nabla_{b} \xi_{c}=2 \lambda g_{c b},
$$

$\lambda$ being a certain function (constant).

Proposition 3.6. In order for a normal variation of a submanifold to be conformal (homothetic), it is necessary and sufficient that

$$
h_{c b x} \xi^{x}=\lambda g_{c b},
$$

$\lambda$ being a certain function (constant), that is, the submanifold is umbilical with respect to the direction of the normal varuation.

COROLlary 1. A submanifold is totally umbilical if and only if every normal variation of the submanifold is conformal.

We denote by $g$ the determinant formed with $g_{c b}$. Then the volume element $d V$ of $M^{n}$ is given by

$$
d V=\sqrt{g} d y^{1} \wedge d y^{2} \wedge \cdots \wedge d y^{n} .
$$

Since we have from (3.1) and (3.2),

we have

$$
\delta \sqrt{g}=\sqrt{g}\left(\nabla_{a} \xi^{a}-h_{a}{ }^{a}{ } \xi^{x}\right) \varepsilon,
$$

$$
\delta d V=\left(\nabla_{a} \xi^{a}-h_{a}{ }^{a}{ }_{x} \xi^{x}\right) d V \varepsilon .
$$

Thus we have

Proposition 3.7. In order for a variation of a submanifold to be volumepreserving, it is necessary and sufficient that

$$
\nabla_{a} \xi^{a}-h_{a}{ }^{a}{ }_{x} \xi^{x}=0 .
$$

PROPOSITION 3.8. [5] In order for a tangential variation of a submanifold to be volume-preserving, it is necessary and sufficient that

$$
\mathcal{L} \sqrt{g}=\nabla_{a} \xi^{a}=0 .
$$

Proposition 3.9. In order for a normal varzation of a submanifold to be volume-preserving, it is necessary and sufficient that 


$$
h_{a}{ }^{a} \xi^{x}=0
$$

that is, the submanifold is minimal with respect to the direction of the normal variation.

COROLLARY 1. A submanifold is minimal if and only if every normal variation of the submanifold is volume-preserving.

\section{$\S 4$. Variations of Christoffel symbols.}

We denote by $\bar{C}_{y}{ }^{n} m-n$ mutually orthogonal unit normals to the deformed submanifold and by $\widetilde{C}_{y}{ }^{h}$ the vectors obtained from $\bar{C}_{y}{ }^{h}$ by parallel displacement of $\bar{C}_{y}{ }^{h}$ from the point $\left(\bar{x}^{h}\right)$ to $\left(x^{h}\right)$. Then we have

$$
\tilde{C}_{y}{ }^{h}=\bar{C}_{y}{ }^{h}+\Gamma_{j i}^{h}(x+\xi \varepsilon) \xi^{\jmath} \bar{C}_{y}{ }^{2} \varepsilon .
$$

We put

$$
\delta C_{y}{ }^{h}=\widetilde{C}_{y}{ }^{h}-C_{y}{ }^{h}
$$

and assume that $\delta C_{y}{ }^{h}$ is of the form

$$
\delta C_{y}{ }^{h}=\eta_{y}{ }^{h} \varepsilon=\left(\eta_{y}{ }^{a} B_{a}{ }^{h}+\eta_{y}{ }^{x} C_{x}{ }^{h}\right) \varepsilon .
$$

Then (4.1), (4.2) and (4.3) give

$$
\bar{C}_{y}{ }^{h}=C_{y}{ }^{h}-\Gamma_{j i}^{h} \xi^{\jmath} C_{y}{ }^{2} \varepsilon+\left(\eta_{y}{ }^{a} B_{a}{ }^{h}+\eta_{y}{ }^{x} C_{x}{ }^{h}\right) \varepsilon .
$$

Applying the operator $\delta$ to $B_{b}{ }^{3} C_{y}{ }^{2} g_{j i}=0$ and using (2.6), (2.11), (4.3) and $\delta g_{j i}=0$, we find

where $\eta_{y b}=\eta_{y}^{c} g_{c b}$, or

$$
\left(\nabla_{b} \xi_{y}+h_{b a y} \xi^{a}\right)+\eta_{y b}=0
$$

$$
\eta_{y}{ }^{a}=-\left(\nabla^{a} \xi_{y}+h_{b}{ }^{a} \xi^{b}\right) .
$$

Applying the operator $\delta$ to $C_{z}{ }^{3} C_{y}{ }^{2} g_{j i}=\delta_{z y}$ and using (4.3) and $\delta g_{j i}=0$, we find

$$
\eta_{y x}+\eta_{x y}=0 \text {, }
$$

where $\eta_{y x}=\eta_{y}{ }^{z} g_{z x}$.

From (4.2) and (4.3), we have

$$
\tilde{C}_{y}{ }^{h}=\left[\eta_{y}{ }^{a} B_{a}{ }^{h}+\left(\delta_{y}^{n}+\eta_{y}{ }^{x}\right) C_{x}{ }^{h}\right] \varepsilon,
$$

which shows that in order that the normal space of the deformed submanifold at the point $\left(\bar{x}^{h}\right)$ and that of the original submanifold at the point $\left(x^{h}\right)$ are parallel, it is necessary and sufficient that $\eta_{y}{ }^{a}=0$, which proves Proposition 2.1.

We denote by $\bar{B}_{\imath}^{\alpha} n$ covectors of the deformed submanifold corresponding to $B^{a}{ }_{\imath}$ of the original submanifold and by $\tilde{B}^{a}{ }_{\imath}$ the covectors obtained from $\bar{B}^{a}{ }_{\imath}$ by parallel displacement of $\bar{B}^{a}{ }_{\imath}$ from the point $\left(\bar{x}^{h}\right)$ to $\left(x^{h}\right)$. Then we have 


$$
\tilde{B}^{a}{ }_{\imath}=\bar{B}_{i}^{a}-\Gamma_{j \iota}^{h}(x+\xi \varepsilon) \xi^{\jmath} \bar{B}_{h}^{a} \varepsilon .
$$

We put

$$
\delta B^{a}{ }_{\imath}=\tilde{B}^{a}{ }_{i}-B^{a}{ }_{\imath} .
$$

Then applying the operator $\delta$ to

$$
B_{b}{ }^{\imath} B^{a}{ }_{\imath}=\delta_{b}^{a}, \quad C_{y}{ }^{\imath} B^{a}{ }_{\imath}=0
$$

and using (2.6) and (4.3), we flnd

$$
\delta B^{a}{ }_{\imath}=-\left(\nabla_{b} \xi^{a}-h_{b}{ }^{a} \xi^{x}\right) B^{b}{ }_{i} \varepsilon+\left(\nabla^{a} \xi_{x}+h_{b}{ }_{x} \xi^{b}\right) C^{x}{ }_{\imath} \varepsilon .
$$

From (4.8), (4.9) and (4.10), we have

$$
\begin{aligned}
\bar{B}^{a}{ }_{i}= & B^{a}{ }_{\imath}+\left[\Gamma_{j i}^{h} \xi^{j} B^{a}{ }_{h}-\left(\nabla_{b} \xi^{a}-h_{b}{ }^{a}{ }_{x} \xi^{x}\right) B^{b}{ }_{\imath}\right. \\
& \left.+\left(\nabla^{a} \xi_{x}+h_{b}{ }^{a}{ }_{x} \xi^{b}\right) C^{x}{ }_{\imath}\right] \varepsilon .
\end{aligned}
$$

We denote by $\bar{C}^{x}{ }_{\imath} m-n$ covectors of the deformed submanifold corresponding to $C^{x}{ }_{2}$ of the original submanifold and by $\widetilde{C}^{x}{ }_{i}$ the covectors obtained from $\bar{C}^{x}{ }_{\imath}$ by parallel displacement of $\bar{C}^{x}{ }_{\imath}$ from the point $\left(\bar{x}^{h}\right)$ to $\left(x^{h}\right)$. Then we have

$$
\widetilde{C}^{x}{ }_{\imath}=\bar{C}^{x}{ }_{\imath}-\Gamma_{j i}^{h}(x+\xi \varepsilon) \xi^{\jmath} \bar{C}_{h}^{x} \varepsilon .
$$

We put

$$
\delta C^{x}{ }_{i}=\widetilde{C}^{x}{ }_{i}-C^{x}{ }_{i} .
$$

Then applying the operator $\delta$ to

$$
B_{b}{ }^{\imath} C^{x}{ }_{\imath}=0, \quad C_{y}{ }^{\imath} C^{x}{ }_{\imath}=\delta_{y}^{x}
$$

and using (2.6) and (4.3), we find

$$
\delta C^{x}{ }_{\imath}=-\left[\left(\nabla_{b} \xi^{x}+h_{b a}{ }^{x} \xi^{a}\right) B^{b}{ }_{\imath}+\eta_{y}{ }^{x} C^{y}{ }_{\imath}\right] \varepsilon .
$$

From (4.12), (4.13) and (4.14), we have

$$
\bar{C}^{x}{ }_{\imath}=C^{x}{ }_{i}+\Gamma_{j i}^{h} \xi^{j} C^{x}{ }_{h} \varepsilon-\left[\left(\nabla_{b} \xi^{x}+h_{b a}{ }^{x} \xi^{a}\right) B^{b}{ }_{\imath}+\eta_{y}{ }^{x} C^{y}{ }_{\imath}\right] \varepsilon .
$$

We now put

$$
\Gamma_{c b}^{a}=\left(\partial_{b} \bar{B}_{b}{ }^{h}+\Gamma_{j i}^{h}(\bar{x}) \bar{B}_{c}{ }^{j} \bar{B}_{b}{ }^{i}\right) \bar{B}^{a}{ }_{h}
$$

and

$$
\delta \Gamma_{c b}^{a}=\bar{\Gamma}_{c b}^{a}-\Gamma_{c b}^{a} .
$$

$\bar{\Gamma}_{c b}^{a}$ are Christoffel symbols of the deformed submanifold.

Substituting (2.2) and (4.11) into (4.16), we obtain by a straightforward computation,

$$
\delta \Gamma_{c b}^{a}=\left[\left(\nabla_{c} \nabla_{b} \xi^{h}+K_{k j i}{ }^{h} \xi^{k} B_{c b}^{j i}\right) B^{a}{ }_{n}+h_{c b}{ }^{x}\left(\nabla^{a} \xi_{x}+h_{d}{ }_{x}{ }_{x} \xi^{d}\right)\right] \varepsilon,
$$


from which, using equations (1.11) of Gauss and those (1.12) of Codazzi,

$$
\begin{aligned}
\delta \Gamma_{c b}^{a}= & \left(\nabla_{c} \nabla_{b} \xi^{a}+K_{d c b}{ }^{a} \xi^{d}\right) \varepsilon \\
& -\left[\nabla_{c}\left(h_{b e x} \xi^{x}\right)+\nabla_{b}\left(h_{c e x} \xi^{x}\right)-\nabla_{e}\left(h_{c b x} \xi^{x}\right)\right] g^{e a} \varepsilon .
\end{aligned}
$$

We now put

$$
\bar{\Gamma}_{c y}^{x}=\left(\partial_{c} \bar{C}_{y}{ }^{h}+\Gamma_{j i}^{h}(\bar{x}) \bar{B}_{c}{ }^{j} \bar{C}_{y}{ }^{i}\right) \bar{C}^{x}{ }_{h}
$$

and

$$
\delta \Gamma_{c y}^{x}=\Gamma_{c y}^{x}-\Gamma_{c y}^{x}
$$

$\bar{\Gamma}_{c y}^{x}$ are components of the connection induced on the normal bundle of the deformed submanifold.

Substituting (2.2), (4.4) and (4.15) into (4.20), we obtain by a straightforward computation

$$
\delta \Gamma_{c y}^{x}=\left[\left(\nabla_{c} \eta_{y}{ }^{h}+K_{k j i}{ }^{h} \xi^{k} B_{c}{ }^{\jmath} C_{y}{ }^{i}\right) C^{x}{ }_{h}+h_{c}{ }^{a}{ }_{y}\left(\nabla_{a} \xi^{x}+h_{a d}{ }^{x} \xi^{d}\right)\right] \varepsilon,
$$

from which, using equations (1.15) of Ricci,

$$
\delta \Gamma_{c y}^{x}=\left[\nabla_{c} \eta_{y}{ }^{x}+K_{d c y}{ }^{x} \xi^{d}+K_{k j i}{ }^{h} C_{z}{ }^{k} B_{c}{ }^{j} C_{y}{ }^{2} C^{x}{ }_{h} \xi^{z}+h_{c}{ }_{y}^{a} \nabla_{a} \xi^{x}-h_{c}{ }^{a x} \nabla_{a} \xi_{y}\right] \varepsilon .
$$

A variation of a submanifold for which $\delta \Gamma_{c b}^{a}=0$ is said to be affine. From (4.19) we have

PROPOSITION 4.1. In order for a variation of a submanifold to be affine, it is necessary and sufficient that

$$
\nabla_{c} \nabla_{b} \xi^{a}+K_{d c b}^{a} \xi^{d}-\left[\nabla_{c}\left(h_{b e x} \xi^{x}\right)+\nabla_{b}\left(h_{c e x} \xi^{x}\right)-\nabla_{e}\left(h_{c b x} \xi^{x}\right)\right] g^{e a}=0 .
$$

COROLLARY 1. [5] In order for a tangential variation of a submanifold to be affine, it is necessary and sufficient that

$$
\mathcal{L} \Gamma_{c b}^{a}=\nabla_{c} \nabla_{b} \xi^{a}+K_{d c b}{ }^{a} \xi^{d}=0 .
$$

For a normal variation of a submanifold we have from (4.19)

$$
\delta \Gamma_{c b}^{a}=-\left[\nabla_{c}\left(h_{b e x} \xi^{x}\right)+\nabla_{b}\left(h_{c e x} \xi^{x}\right)-\nabla_{e}\left(h_{c b x} \xi^{x}\right)\right] g^{e a} \varepsilon,
$$

from which

$$
\nabla_{c}\left(h_{b a x} \xi^{x}\right) \varepsilon=-\frac{1}{2}\left[\left(\delta \Gamma_{c b}^{e}\right) g_{e a}+\left(\delta \Gamma_{\iota a}^{e}\right) g_{e b}\right] .
$$

From (4.26) and (4.27), we have

COROLlaRY 2. In order for a normal variation of a submanifold to be affine, it is necessary and sufficient that

$$
\nabla_{c}\left(h_{b a x} \xi^{x}\right)=0,
$$

that is, the second fundamental form with respect to the variation vector is parallel. 
CORCllaRY 3. If a submanifold with parallel second fundamental tensors admits a parallel normal variation, then it is affine.

For a compact orientable submanifold $M^{n}$, we have the following integral formula :

$$
\int\left[\left(g^{c b} \nabla_{c} \nabla_{b} \xi^{a}+K_{d}^{a} \xi^{d}\right) \xi_{a}+\frac{1}{2}\left(\nabla_{c} \xi_{b}+\nabla_{b} \xi_{c}\right)\left(\nabla^{c} \xi^{b}+\nabla^{b} \xi^{c}\right)-\left(\nabla_{b} \xi^{b}\right)^{2}\right] d V=0,
$$

which is valid for any vector field $\xi^{a}$ in $M^{n}[6]$. From (4.29), we find

$$
\begin{aligned}
& \int\left[\left\{g^{c b} \nabla_{c} \nabla_{b} \xi^{a}+K_{d}{ }^{a} \xi^{d}-2 \nabla^{c}\left(h_{c}{ }^{a}{ }_{x} \xi^{x}\right)+\nabla^{a}\left(h_{b}{ }^{b}{ }_{x} \xi^{x}\right)\right\} \xi_{a}\right. \\
& \quad+\frac{1}{2}\left(\nabla_{c} \xi_{b}+\nabla_{b} \xi_{c}-2 h_{c b y} \xi^{y}\right)\left(\nabla^{c} \xi^{b}+\nabla^{b} \xi^{c}-2 h^{c b}{ }_{x} \xi^{x}\right) \\
& \quad-\left(\nabla_{c} \xi^{c}-h_{c}{ }^{c} \xi^{x}\right)\left(\nabla_{b} \xi^{b}\right) \\
& \left.\quad+\left(\nabla_{c} \xi_{b}+\nabla_{b} \xi_{c}-2 h_{c b y} \xi^{y}\right) h^{c b}{ }_{x} \xi^{x}\right] d V=0 .
\end{aligned}
$$

Now if a variation of the submanifold is isometric, we have (3.3) and consequently

$$
\left(\nabla_{c} \xi_{b}+\nabla_{b} \xi_{c}-2 h_{c b y} \xi^{y}\right) h^{c b} \xi^{x}=0 \text { and } \nabla_{c} \xi^{c}-h_{c}{ }^{c} \xi^{x}=0
$$

Since an isometric variation is affine, we also have (4.24), from which

$$
g^{c b} \nabla_{c} \nabla_{b} \xi^{a}+K_{d}{ }^{a} \xi^{d}-2 \nabla^{c}\left(h_{c}{ }^{a}{ }_{x} \xi^{x}\right)+\nabla^{a}\left(h_{b}{ }^{b}{ }_{x} \xi^{x}\right)=0 .
$$

Conversely if (4.31) and (4.32) are satisfied, we have from (4.30),

$$
\nabla_{c} \xi_{b}+\nabla_{b} \xi_{c}-2 h_{c b x} \xi^{x}=0
$$

and consequently the variation is isometric. Thus we have

Proposition 4.2. In order for a variation of a compact submanifold to be isometric, it is necessary and sufficient that we have (4.31) and (4.32).

Now, from (4.24), we have

$$
\nabla_{c} \nabla_{b} \xi_{a}+K_{a c b a} \xi^{d}-\nabla_{c}\left(h_{b a x} \xi^{x}\right)+\nabla_{b}\left(h_{c a x} \xi^{x}\right)-\nabla_{a}\left(h_{c b x} \xi^{x}\right)=0
$$

$K_{d c b a}$ being covariant components of the curvature tensor of $M^{n}$, from which using the identity $K_{d c b a}+K_{d c a b}=0$,

$$
\nabla_{c}\left(\nabla_{b} \xi_{a}+\nabla_{a} \xi_{b}-2 h_{b a x} \xi^{x}\right)=0 .
$$

Thus if the submanifold is complete and irreducible, we have

$$
\nabla_{b} \xi_{a}+\nabla_{a} \xi_{b}-2 h_{b a x} \xi^{x}=2 \lambda g_{b a},
$$


$\lambda$ being a constant.

Conversely from (4.34) we can deduce (4.33) which is equivalent to (4.24). Thus we have

Proposition 4.3. A variation of a complete and irreducible submantfold is affine if and only if it is homothetic.

From (4.34), we have

COROLLARY 1. If a complete and rrreducible submanifold admits an affine normal variation, then the submanifold is umbilical with respect to the variation vector.

\section{$\S 5$. Variations of the second fundamental tensors.}

Suppose that $v^{h}$ is a vector field of $M^{m}$ defined intrinsically along the submanifold $M^{n}$. When we displace the submanifold $M^{n}$ by $\bar{x}^{h}=x^{h}+\xi^{h}(y) \varepsilon$ in the direction $\xi^{h}$, we obtain a vector field $\tilde{v}^{h}$ which is defined also intrinsically along the deformed submanifold. If we displace $\bar{v}^{h}$ back parallelly from the point $\left(\bar{x}^{h}\right)$ to $\left(x^{h}\right)$, we obtain

$$
\tilde{v}^{h}=\bar{v}^{h}+\Gamma_{j i}^{h}(x+\xi \varepsilon) \xi^{j} \bar{v}^{2} \varepsilon
$$

and hence forming

$$
\delta v^{h}=\tilde{v}^{h}-v^{h},
$$

we find

$$
\delta v^{h}=\bar{v}^{h}-v^{h}+\Gamma_{j i}^{h} \xi^{\jmath} v^{\imath} \varepsilon .
$$

Similarly we have

$$
\delta \Gamma_{c} v^{h}=\bar{\nabla}_{c} \bar{v}^{h}-\nabla_{c} v^{h}+\Gamma_{j i}^{h} \xi^{j} \nabla_{c} v^{2} \varepsilon,
$$

that is,

$$
\begin{aligned}
\delta V_{c} v^{h}= & \nabla_{c} \bar{v}^{h}-\nabla_{c} v^{h}+\left(\partial_{k} \Gamma_{j i}^{h}+\Gamma_{k t}^{h} \Gamma_{j i}^{t}\right) \xi^{k} B_{c}{ }^{\jmath} v^{2} \varepsilon \\
& +\Gamma_{j i}^{h}\left[\left(\partial_{c} \xi^{j}\right) v^{2}+\xi^{\jmath}\left(\partial_{c} v^{i}\right)\right] \varepsilon .
\end{aligned}
$$

On the other hand, from (5.2) we have

$$
\begin{aligned}
\nabla_{c} \delta v^{h}= & \nabla_{c} \bar{v}^{h}-\nabla_{c} v^{h}+\left(\partial_{j} \Gamma_{k z}^{h}+\Gamma_{j t}^{h} \Gamma_{k i}^{t}\right) \xi^{k} B_{c}{ }^{j} v^{2} \varepsilon \\
& +\Gamma_{j i}^{h}\left[\left(\partial_{c} \xi^{j}\right) v^{2}+\xi^{\jmath}\left(\partial_{c} v^{2}\right)\right] \varepsilon .
\end{aligned}
$$

Thus forming (5.3)-(5.4), we find

$$
\delta \nabla_{c} v^{h}-\nabla_{c} \delta v^{h}=K_{k j i}{ }^{h} \xi^{k} B_{c}{ }^{j} v^{\imath} \varepsilon .
$$

Similarly, for a covector $w_{\imath}$, we have 


$$
\delta \nabla_{c} w_{\imath}-\nabla_{c} \delta w_{\imath}=-K_{k j i}{ }^{h} \xi^{k} B_{c}{ }^{j} w_{h} \varepsilon .
$$

For a tensor field carrying three kinds of indices, say, $T_{b y}{ }^{h}$, we have

$$
\begin{aligned}
\delta V_{c} T_{b y}{ }^{h} & -\nabla_{c} \delta T_{b y}{ }^{h} \\
& =K_{k j i}{ }^{h} \xi^{k} B_{c}{ }^{j} T_{b y}{ }^{2} \varepsilon-\left(\delta \Gamma_{c b}^{a}\right) T_{a y}{ }^{h}-\left(\delta \Gamma_{c y}^{x}\right) T_{b x}{ }^{h} .
\end{aligned}
$$

Applying the formula (5.7) to $B_{b}{ }^{h}$, we find

$$
\delta V_{c} B_{b}{ }^{h}-\nabla_{c} \delta B_{b}{ }^{h}=K_{k j i}{ }^{h} \xi^{k} B_{c}{ }^{j} B_{b}{ }^{2} \varepsilon-\left(\delta \Gamma_{c b}^{a}\right) B_{a}{ }^{h},
$$

or using (1.9) and (2.6)

$$
\delta\left(h_{c b}{ }^{x} C_{x}{ }^{h}\right)=\left(\nabla_{c} \nabla_{b} \xi^{h}+K_{k \jmath 2}{ }^{h} \xi^{k} B_{c}{ }^{\jmath} B_{b}{ }^{i}\right) \varepsilon-\left(\delta \Gamma_{c b}^{a}\right) B_{a}{ }^{h},
$$

from which, using (4.3),

$$
\begin{aligned}
& \left(\delta h_{c b}{ }^{x}\right) C_{x}{ }^{h}+h_{c b}{ }^{x}\left(\eta_{x}{ }^{a} B_{a}{ }^{h}+\eta_{x}{ }^{y} C_{y}{ }^{h}\right) \varepsilon \\
& =\left(\nabla_{c} \nabla_{b} \xi^{h}+K_{k j z}{ }^{h} \xi^{k} B_{c}{ }^{j} B_{b}{ }^{i}\right) \varepsilon-\left(\delta \Gamma_{c b}^{a}\right) B_{a}{ }^{h} .
\end{aligned}
$$

Thus

$$
\delta h_{c b}{ }^{x}=-h_{c b}{ }^{y} \eta_{y}{ }^{x} \varepsilon+\left(\nabla_{c} \nabla_{b} \xi^{h}+K_{k j i}{ }^{h} \xi^{k} B_{c}{ }^{j} B_{b}{ }^{i}\right) C^{x}{ }_{h} \varepsilon,
$$

from which

$$
\begin{aligned}
\delta h_{c b}{ }^{x}= & {\left[\xi^{d} \nabla_{d} h_{c b}{ }^{x}+h_{e b}{ }^{x}\left(\nabla_{c} \xi^{e}\right)+h_{c e}{ }^{x}\left(\nabla_{b} \xi^{e}\right)-h_{c b}{ }^{y} \eta y_{y}{ }^{x}\right] \varepsilon } \\
& +\left[\nabla_{c} \nabla_{b} \xi^{x}+K_{k j i}{ }^{h} C_{y}{ }^{k} B_{c b}^{j i} C^{x}{ }_{h} \xi^{y}-h_{c e}{ }^{x} h_{b}{ }^{e} \xi^{y}\right] \varepsilon .
\end{aligned}
$$

Thus we have

Proposition 5.1. A variation of a submanifold gives the variation (5.8) to the second fundamental forms and consequently it preserves the second fundamental forms if and only if

$$
\begin{aligned}
& {\left[\xi^{d} \nabla_{d} h_{c b}{ }^{x}+h_{e b}{ }^{x}\left(\nabla_{c} \xi^{e}\right)+h_{c e}{ }^{x}\left(\nabla_{b} \xi^{e}\right)-h_{c b}{ }^{y} \eta_{y}{ }^{x}\right]} \\
& +\left[\nabla_{c} \nabla_{b} \xi^{x}+K_{k j i}{ }^{h} C_{y}{ }^{k} B_{c b}^{j i} C^{x}{ }_{h} \xi^{y}-h_{c e}{ }^{x} h_{b}{ }^{e}{ }_{y} \xi^{y}\right]=0 .
\end{aligned}
$$

Proposition 5.2. For a tangential variation of a submanifold, we have

$$
\delta h_{c b}{ }^{x}=\left[\xi^{d} \nabla_{d} h_{c b}{ }^{x}+h_{e b}{ }^{x}\left(\nabla_{c} \xi^{e}\right)+h_{c e}{ }^{x}\left(\nabla_{b} \xi^{e}\right)-h_{c b}{ }^{y} \eta_{y}{ }^{x}\right] \varepsilon
$$

and consequently a tangential variation of a submanifold preserves the second fundamental forms if and only if

$$
\xi^{d} \nabla_{d} h_{c b}{ }^{x}+h_{e b}{ }^{x}\left(\nabla_{c} \xi^{e}\right)+h_{c e}{ }^{x}\left(\nabla_{b} \xi^{e}\right)-h_{c b}{ }^{y} \eta_{y}{ }^{x}=0 .
$$

Proposition 5.3. [2] For a normal variation of a submanifold, we have

$$
\delta h_{c b}{ }^{x}=\left[\nabla_{c} \nabla_{b} \xi^{x}+K_{k j i}{ }^{h} C_{y}{ }^{k} B_{c b}^{j i} C^{x}{ }_{h} \xi^{y}-h_{c e}{ }^{x} h_{b}{ }^{e} \xi^{y}-h_{c b}{ }^{y} \eta_{y}{ }^{x}\right] \varepsilon
$$


and consequently a normal variation of a submanfold preserves the second fundamental forms if and only if

$$
\nabla_{c} \nabla_{b} \xi^{x}+K_{k j i}{ }^{h} C_{y}{ }^{k} B_{c b}^{j i} C^{x}{ }_{h} \xi^{y}-h_{c e}{ }^{x} h_{b}{ }^{e}{ }_{y} \xi^{y}-h_{c b}{ }^{y} \eta_{y}{ }^{x}=0 .
$$

COROLlary 1. [2] A normal variation carries a totally geodesic submanifold into a totally geodesic submanfold if and only if

$$
\nabla_{c} \nabla_{b} \xi^{x}+K_{k j i}{ }^{h} C_{y}{ }^{k} B_{i b}^{j i} C^{x}{ }_{h} \xi^{y}=0 .
$$

COROLlARY 2. [2] A normal variation carries a totally umbilical submanifold into a totally umbilical submanifold if and only if

$$
\nabla_{c} \nabla_{b} \xi^{x}+K_{k j i}{ }^{h} C_{y}{ }^{k} B_{c b}^{j i} C^{x}{ }_{h} \xi^{y}=g_{c b} \alpha^{x},
$$

$\alpha^{x}$ being certain functions.

Since for a normal variation we have from (3.2)

that is,

$$
\delta\left(g^{c b} h_{c b}{ }^{x}\right)=\left(\delta g^{c b}\right) h_{c b}{ }^{x}+g^{c b} \delta\left(h_{c b}{ }^{x}\right),
$$

$$
\delta\left(g^{c b} h_{c b}{ }^{x}\right)=2 h^{c b} \xi^{y} h_{c b}^{x}+g^{c b} \delta\left(h_{c b}^{x}\right),
$$

we obtain from (5.12)

$$
\begin{aligned}
\delta\left(\frac{1}{n} g^{c b} h_{c b}{ }^{x}\right)= & \frac{1}{n}\left[g^{c b} \nabla_{c} \nabla_{b} \xi^{x}+K_{k j i}{ }^{h} C_{y}{ }^{k} B^{j i} C^{x}{ }_{h} \xi^{y}\right. \\
& \left.+h_{c b}{ }^{x} h^{c b}{ }_{y} \xi^{y}-h_{a}{ }^{a y} \eta_{y}{ }^{x}\right] \varepsilon,
\end{aligned}
$$

where $B^{j i}=B_{c b}^{j i} g^{c b}$.

Thus we have

PROPOSITION 5.4. For a normal variatıon of a submanifold, we have (5.16) and consequently a normal variation preserves the mean curvature vector if and only if

$$
g^{c b} \nabla_{c} \nabla_{b} \xi^{x}+K_{k j i}{ }^{h} C_{y}{ }^{k} B^{j i} C^{x}{ }_{h} \xi^{y}+h_{c b}{ }^{x}{ }^{c b}{ }_{y} \xi^{y}-h_{a}{ }^{a y} \eta_{y}{ }^{x}=0 .
$$

COROLlary 1. [2] A normal variation carries a minımal submanifold into a minimal submanifold if and only if

$$
g^{c b} \nabla_{c} \nabla_{b} \xi^{x}+K_{k j i}{ }^{h} C_{y}{ }^{k} B^{j i} C^{x}{ }_{h} \xi^{y}+h_{c b}{ }^{x}{ }^{c b}{ }_{y} \xi^{y}=0 .
$$

Suppose that a normal variation carries a minimal submanifold into a minimal submanifold. Then substituting (5.18) into

$$
\frac{1}{2} \Delta\left(\xi^{x} \xi_{x}\right)=\frac{1}{2} g^{c b} \nabla_{c} \nabla_{b}\left(\xi^{x} \xi_{x}\right)=\left(g^{c b} \nabla_{c} \nabla_{b} \xi^{x}\right) \xi_{x}+\left(\nabla_{c} \xi_{x}\right)\left(\nabla^{c} \xi^{x}\right)
$$

we find 


$$
\begin{aligned}
\frac{1}{2} \Delta\left(\xi^{x} \xi_{x}\right)= & -K_{k j i h} C_{y}{ }^{k} B^{j i} C_{x}{ }^{h} \xi^{y} \xi^{x} \\
& -\left(h_{c b y} \xi^{y}\right)\left(h^{c b}{ }_{x} \xi^{x}\right)+\left(\nabla^{c} \xi^{x}\right)\left(\nabla_{c} \xi_{x}\right) .
\end{aligned}
$$

Now suppose that a parallel normal variation carries a minimal submanifold in

$$
K_{k j i h} C_{y}{ }^{k} B^{j i} C_{x}{ }^{n} \xi^{y} \xi^{x}+\left(h_{c b y} \xi^{y}\right)\left(h^{c b}{ }_{x} \xi^{x}\right)=0 .
$$

Thus if the sectional curvature of $M^{m}$ with respect to the section spanned by the variation vector and a tangent to the submanifold is non-positive, we have

$$
-K_{k j i n} C_{y}{ }^{k} B^{j i} C_{x}{ }^{h} \xi^{y} \xi^{x} \leqq 0,
$$

and consequently from (5.20)

$$
h_{c b x} \xi^{x}=0
$$

Thus we have

PROPOSITION 5.5. If a parallel normal variation corries a minmal submanfold into a minmal submanifold and the sectional curvature of the ambiant manifold with respect to a section spanned by the variation vector and a tangent to the submanifold is non-positive, then the submanifold is geodesic with respect to the variation vector.

We now consider a normal variation $\bar{x}^{h}=x^{h}+\lambda C^{h} \varepsilon$ of a compact hypersurface, where $\lambda$ is a positive function and $C^{h}$ the unit normal to the hypersurface.

In this case (3.2) reduces to

$$
\delta g^{c b}=2 \lambda h^{c b} \varepsilon
$$

and (5.12) to

$$
\delta h_{c b}=\left[\nabla_{c} \nabla_{b} \lambda+\lambda K_{k j i h} C^{k} B_{c b}^{j i} C^{h}-\lambda h_{c e} h_{b}{ }^{e}\right] \varepsilon,
$$

$\eta_{y}{ }^{x}$ being identically zero. Thus from (5.21) and (5.22) we have

$$
\delta\left(g^{c b} h_{c b}\right)=\left[\Delta \lambda+\lambda K_{k j i h} C^{k} B^{j i} C^{h}+\lambda h_{c b} h^{c b}\right] \varepsilon .
$$

Thus if the normal variation preserves $g^{c b} h_{c b}$ we have

$$
\Delta \lambda+\lambda\left[K_{k j i h} C^{k} B^{j i} C^{h}+h_{c b} h^{c b}\right]=0 .
$$

Consequently if moreover

$$
K_{k j i h} C^{k} B^{j \imath} C^{h} \geqq 0,
$$

we have

$$
\lambda=\text { constant }, \quad K_{k j 2 h} C^{k} B^{j i} C^{h}=0 \text { and } \quad h_{c b}=0 .
$$

Thus we have 
Proposition 5.6. If a normal variation $\bar{x}^{h}=x^{h}+\lambda C^{h} \varepsilon, \lambda>0$, of a compact hypersurface preserves $g^{c b} h_{c b}$ and $K_{k j i h} C^{k} B^{j i} C^{h} \geqq 0$, then we have

$$
\lambda=\text { constant, } \quad K_{k j i h} C^{k} B^{j i} C^{h}=0
$$

and the hypersurface is totally geodesic.

\section{BIBLIOGRAPHY}

[1] Bang-Yen Chen, Geometry of submanıfolds, Marcel Dekker, Inc., New York (1973).

[2] BANG-Yen Chen AND K. YANO, On the theory of normal variations, to appear.

[3] J. A. Schouthn, Ricci-Calculus, Springer (1954).

[4] K. YANO, Sur la théorie des déformations infinitésimales, J. of the Fac. of Sci. Univ. of Tokyo, 6 (1949), 1-75.

[5] K. YANO, The theory of Lie derivatives and 1ts applications, North-Holland Publ. Co., Amsterdam (1957).

[6] K. YANo, Integral formulas in Riemannıan geometry, Marcel Dekker, Inc., New York (1970).

TOKyo Institute of TECHNOLOgy 